\title{
Cyril Blet, Une voix mondiale pour un État. France 24
}

Paris, Éd. L'Harmattan, 2008

\section{Gérald Arboit}

\section{OpenEdition}

\section{Journals}

Édition électronique

URL : http://journals.openedition.org/questionsdecommunication/718

DOI : 10.4000 /questionsdecommunication.718

ISSN : 2259-8901

\section{Éditeur}

Presses universitaires de Lorraine

\section{Édition imprimée}

Date de publication : 1 juillet 2009

Pagination : 388-389

ISBN : 978-2-86480-989-0

ISSN : $1633-5961$

\section{Référence électronique}

Gérald Arboit, "Cyril Blet, Une voix mondiale pour un État. France 24 », Questions de communication [En ligne], 15 | 2009, mis en ligne le 13 janvier 2012, consulté le 22 septembre 2020. URL : http:// journals.openedition.org/questionsdecommunication/718; DOI : https://doi.org/10.4000/ questionsdecommunication.718

Ce document a été généré automatiquement le 22 septembre 2020

Tous droits réservés 


\title{
Cyril Blet, Une voix mondiale pour un État. France 24
}

Paris, Éd. L'Harmattan, 2008

\author{
Gérald Arboit
}

\section{RÉFÉRENCE}

Cyril Blet, Une voix mondiale pour un État. France 24. Paris, Éd. L'Harmattan, coll. Chaos international, 2008, $238 \mathrm{p}$.

1 Être le premier ne signifie pas pour autant être le meilleur. Tel pourrait être caractérisé le livre de Cyril Blet. Même réactualisé pour la publication et agrémenté d'une longue préface d'Hervé Bourges (pp. 11-30), il reste ce mémoire de master recherche qu'il était lorsqu'il a été soutenu à l'université Paris I Panthéon-Sorbonne. Bien construit autour de deux parties (« Une diplomatie planétaire de l'information », pp. 51-141; « Une voix globalisée de la France ", pp. 143-209) et quatre chapitres, il se nourrit de nombreuses références, dont les plus récentes datent d'avril 2008, et d'entretiens d'acteurs mondiaux, nationaux et de la chaîne, menés entre le 27 mars et le 27 septembre 2007. Parmi ceux- ci, se trouve Georges-Marc Benamou, encore conseiller culturel à la Présidence et inspirateur, deux mois plus tard, avec son collègue diplomate, Jean-David Lévitte, d'un rapport sur la réforme de l'audiovisuel extérieur français. Immanquablement, le jeune étudiant, qui effectuait à l'époque un stage dans les services du Premier ministre, n'a pas su conserver une distance avec son objet et s'est fait, au travers de sa problématique, le porte-parole de "France Monde », la nouvelle holding censée rationaliser l'audiovisuel extérieur.

D'emblée, l'auteur se place dans la perspective d'une lutte mondiale pour «limiter la puissance de l'hegemon américain et simultanément [...] promouvoir la multipolarité ou encore [...] atténuer les capacités d'agir des États-Unis pour accroître sa propre puissance " (p. 43). Ainsi passe-t-il en revue les différentes chaînes internationales d'information pour mieux se placer dans un va-et-vient comparatif entre CNN, BBC 
World, Deutsche Welle et France 24 des plus improductif. Tout au plus parvient-il à démontrer ce que le jeune canal français n'est pas... On notera cependant qu'elle n'est jamais comparée à Al Jazeera qui, dans la chronologie et dans l'objet, est somme toute plus proche de France 24 que CNN, ni à Euronews ou TV5, qui sont, dans le cas d'espèce franco-français, sur le même créneau. De même part-il à la recherche de la réalité d'un " point de vue français » ou d'un marché publicitaire international. Dans le premier cas, il néglige l'apport de Dominique Marchetti (En quête d'Europe: médias européens et médiatisation de l'Europe, Rennes, Presses universitaires de Rennes, 2004),pourtant cité dans la bibliographie, qui a démontré, sur l'exemple d'Euronews, comment il était d'abord postulé. Quant à la publicité internationale de haut de gamme, un recours au travail de Simona di Iulio ( La publicité transnationale entre universalisme marchand et ancrage territorial ", Les Enjeux de l'information et de la communication, 2002, http:// halshs.archives-ouvertes.fr/docs/00/06/22/53/PDF/sic_00000647.pdf facilement accessible, aurait permis d'apporter un élément de réponse qui, confronté à l'audience espérée de France 24, comme des autres chaînes internationales d'information d'ailleurs, aurait permis de souligner la difficulté de trouver des produits haut de gamme. À défaut de cette démarche scientifique, le visionnage des écrans publicitaires des différentes chaînes citées aurait suffi à permettre un constat. D'un point de vue plus conceptuel, à trop manier la comparaison, l'auteur se montre souvent dérouté par son objet (e.g. p. 55), qu'il a mal cerné en se refusant à traiter de la genèse de la chaîne. Ainsi n'accorde-t-il aucun traitement à la question sociale alors qu'il décrit la rédaction (pp. 169-171). Mais il ne parvient pas plus à trancher, voire affirmant tout et son contraire d'une page à l'autre (e.g. pp. 102 et 106, 99 et 128, 147 et 148, 158 et 161...), quant ce n'est pas à quelques lignes de distance (p. 201). Ainsi ne comprend-il pas vraiment la mutation des Observateurs qui, mieux que de "donner un rôle actif au public dans le processus de collecte des informations » (p. 202), est un véritable moyen de traitement live de l'actualité autant que de suppléer à la faiblesse du réseau de correspondants de France 24. De même, ce passage par l'internet de la chaîne correspond, depuis le premier jour de son lancement en décembre 2006, à un phénomène croissant des télévisions, généralistes ou thématiques.

Il ressort de la lecture de ce mémoire une vision gaullienne de bazar, teintée d'un antigaullisme le plus primaire. Dans un premier temps, il est tout à fait dans la ligne de la pensée de Benamou, comme lorsqu'il fait, le plus sérieusement du monde, de «l'État français » le « chef de file mondial des politiques de diversité culturelle » (p. 197)! Dans un second, il fait écho aux débats sur la chaîne qui se retrouvaient dans la presse au moment de la rédaction du mémoire, comme lorsque, plus tôt, il avait glosé sur la "construction de l'autonomie de France 24 » pour «se détacher de l'image d'une chaîne sous tutelle étatique" (p. 86). Ce faisant, en accumulant maladresses et incompréhensions évidentes, le livre rate son intérêt affirmé pourtant en introduction, mettre «à jour la pratique d'une diplomatie planétaire de l'information» (p.46). En comparant les spécificités de chacune des chaînes internationales d'information, il aurait pu établir une géopolitique de l'information, distinguant les différents marchés sur lesquelles elles interviennent (publicitaires, information locale ou nationale, anglophonie), plutôt que de placer cela sur le compte d'« une rivalité entre acteurs étatiques [...], entités régionales [...] et firmes » (p. 68). Si le "média est le message ", comme le disait Marshall McLuhan, ce n'est pas dans "une approche d'économie politique internationale » qu'il se situe, mais dans son positionnement et son affichage. En matière de chaînes internationales d'information, seule la crise permet de préciser 
si elles possèdent "une voix mondiale ». En ce qui concerne France 24, il est encore trop tôt pour l'affirmer. Et si l'on regarde les bases sur lesquelles elle est bâtie, il est à craindre qu'elle ne l'obtienne jamais.

\section{AUTEURS}

GÉRALD ARBOIT

CERE, Luxembourg

gerald.arboit@aliceadsl.fr 